\author{
DFPARTAMH:NO DE ANATOMIA DESCRITIVA DOS ANIMIS DOMHSTICOS \\ Iirctor: Prof. Dr. Orlando M. Paiva
}

\title{
IRREGULARIDADES DA ANSA SPIRALIS DO CÓLON EM CAPRINOS *
}

(IRREGULAR PATTERNS OF THE ANSA SPIRALIS OF THE
GOAT COI.ON)

VICENTE: BORK:LLI Instrutor A.vtonio Fernandes Fililo
Instrutor

A ocorrência de irregularidades na disposição da ansa spiralis, que juntamente com a ansa proximalis e a ansa distalis forma o colon primum, homólogo do colon ascendens do homem, parece não constituir condição excepcional nos ruminantes domésticos.

De fato, Ellenberger e Baudi (1932) e Martin e Schauder (193.) ) aludem, em suas obras, à possibilidade de aparecerem imperfeiçōes nesse segmento intestinal.

Por outro lado, dentre as publicaçōes especiais relativas a caprinos, KoldA (1931) assinala falha de conformação em 1 dos 4 casos examinados; SuITH (1959) separa, de 50 exemplares, 6 cỏlons defeituosamente enrolados; NANDA e PATEL (1962) reportam 215 incorreçōes nas vísceras dos 1136 espécimes investigados; enfim, PAIVA e BORFilli (1965), ao evidenciarem vícios de configuração em 70 das 946 peças submetidas a exame, deixam-nas de parte, imersas em solução formolada, para ulterior estudo, ora levado a têrmo, pôsto que preocupados somente com o comportamento das regulares, deparadas a descreverem dois e meio e três, três e três e meio, três e meio e quatro e quatro e quatro e meio gyri, respectivamente, centripetales e centrifugales.

Aliás, êstes AA. (1963/64), cuidando dos arranjos incomuns da ansa spiralis de bovinos azebuados, seguem critério de classificação diverso do adotado por SMith (1958), para ovinos. Com efeito, preferem considerar os desvios como se inseridos estivessem em cólons normais, a distribuí-los por três categorias, consoante

\footnotetext{
- Comunlcado a XVIII Conferennia Anual da Sociedade paulista de Medicina veterinaria em setembro de 1963 e apresentado ao IX Congresso Brasileiro de Medicina Veterinaria (Salvador, Bahia) em Julho de 1964.

Trabalho realizado sob) os aluspicios da Fundacão de Amparo a Pesquisa do Fistado de São Palulo.
} 
localizados apenas na volta final, sôbre giro que não o derradeiro e simultâneamente no lance de saida, onde SMITH (1955), descobre cêrca de 25'; dêles, e em qualquer outra secção da espiral. Cabe aqui lembrar, a propósito, Siırth e Meadows (1956) quando dāo como livre de irregularidades a última curva distal do cólon de bovinos.

Como se adiantou, utilizamo-nos de $70\left(7,39 \% \pm 0,85^{*}\right)$, de um grupo de 946 cólons, separados de $46(4,86 \% \pm 0,69)$ machos (m) e $24(2,54 \% \pm 0,51)$ fêmeas (f), de várias idades e raças não definidas. Demonstrada não significante a diferença de incidência entre os sexos, fizemos esquemas de todos os casos, analisando-os conjuntamente e tipificando-os à maneira PAIVA e Borblli (figs. 1 a 40 ).
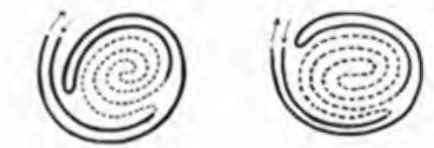

1-obs. 1.2 e 3

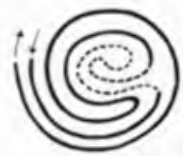

6-obs. 8

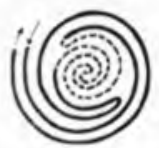

11-0bs. 14 e 15

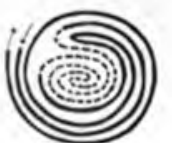

$16-0$ bs. 24 e 25

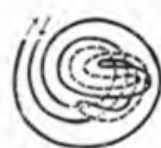

21-Obs. 30
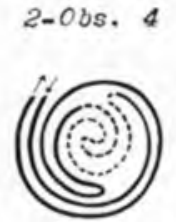

7-obs. 9

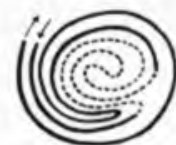

12-Obs. 16

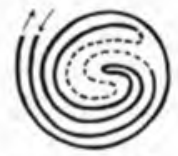

17-Obs. 26

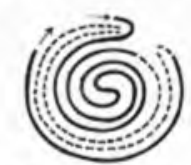

22-Obs. 31
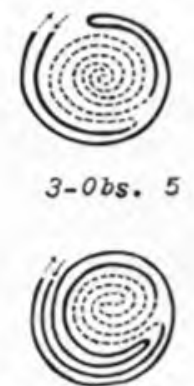

8-0bs. 10 e 11

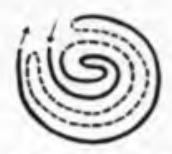

13-Obs. 17
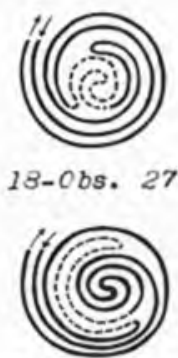

23-obs.32, 33, $34,35,36$ e 37

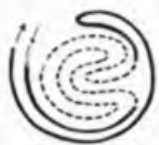

4-Obs. 6

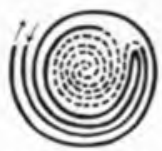

9-Obs. 12

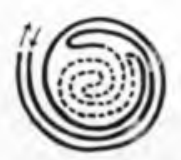

14-Obs, 18

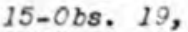

$20,21,22=23$

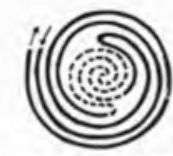

19-Obs. 28

20-Obs. 29
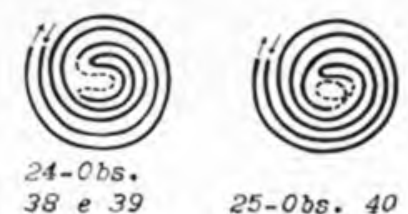

25-Obs. 40

Figuras 1 a 25 - Esquemas das disposicōes incomuns da ansa sprirulis do cólon, em caprinos.

\footnotetext{
- desvio pacirão
} 
Assim, as imperfeiçōes encontradas nos cólons com uma espira de entrada e uma e meia de saída **, inscrevem-se sempre sôbre as meias voltas concêntricas, ou seja, 5 vêzes entre a primeira c a segunda (Obs. $1 \mathrm{~m}, 2 \mathrm{~m}, 3 \mathrm{~m}, 4 \mathrm{~m}, 5 \mathrm{~m}$ ) e 2 , na segunda (Obs. $6 \mathrm{~m}$, $7 \mathrm{~m}$ ). Os defeitos registrados nas peças com um e meio e dois circuitos*, respectivamente proximais e distais, acham-se 9 vêzes nas meias voltas concêntricas, isto é, 5 na segunda (Obs. $8 \mathrm{~m}, 9 \mathrm{f}$, $10 \mathrm{~m}, 11 \mathrm{~m}, 12 \mathrm{~m}$ ), 3 entre esta e a terceira (Obs. $13 \mathrm{~m}, 14 \mathrm{f}, 15 \mathrm{f})$ e 1 vez entre a primeira e a segunda (Obs. 16m). Em 1 caso (Obs. 17f), a incorreção situa-se no terceiro meio giro excêntrico, noutro (Obs. $18 \mathrm{~m}$ ) vêmo-la postada na flexura centralis. As falhas idenlificadas em alças com dois lances a avizinharem-se do centro e dois e meio a afastarem-se dêle **, colocam-se 10 vêzes nas meias voltas concêntricas, a saber, 7 entre a segunda e a terceira (Obs. $19 \mathrm{~m}, 20 \mathrm{~m}$, $21 \mathrm{~m}, 22 \mathrm{~m}, 23 \mathrm{r}, 24 \mathrm{~m}, 25 \mathrm{~m}), 1$ vez na segunda $(O b s .26 \mathrm{~m}), 1$ na terceira (Obs. 27m) e 1 entre a terceira e a quarta (Obs. 28m). Ėm 3 cólons, os vicios de comportamento instalam-se nas meias curvas excêntricas, mais exatamente, $1 \mathrm{vez}$ entre a segunda e a terccira (Obs. 29m), 1 na terceira (Obs. 30f) e 1 na quarta (Obs. 31f). As espiras com dois e meio e três, três e três e meio, três e meio e quatro, quatro e quatro e meio giros, por ordem, centrípetos e centrifugos, mostram, afora uma exceção, incorreçōes nas meias voltas excêntricas. Encontramo-las a incidirem sôbre as do primeiro tipo, 9 vêzes: 6 entre a terceira e a quarta (Obs. $32 \mathrm{~m}$, $33 \mathrm{~m}, 34 \mathrm{~m}, 35 \mathrm{~m}, 36 \mathrm{~m}, 37 \mathrm{~m}), 2$ na segunda (Obs. $38 \mathrm{~m}, 39 \mathrm{f}$ ) e 1 vez entre a primeira e a segunda (Obs. 40f); 8 vêzes, sôbre as do segundo: 3 na sexta (Obs. 41m, 42m, 431), 2 na sẻtima (Obs. 44f, $45 \mathrm{f}$ ), 1 vez na quarta (Obs. 46f), 1 entre a quarta e a quinta (Obs. $47 \mathrm{~m})$ e 1 na quinta (Obs. $48 \mathrm{~m}$ ); 12 vêzes, sôbre as do terceiro: 9 na sétima (Obs. $49 \mathrm{~m}, 50 \mathrm{~m}, 51 \mathrm{~m}, 52 \mathrm{f}, 53 \mathrm{f}, 54 \mathrm{f}, 55 \mathrm{~m}, 56 \mathrm{~m}, 57 \mathrm{f}$ ), 1 vez na sexta (Obs. 59f), 1 entre a sétima e a oitava (Obs. 60f) e 1 na quinta e na sétima, simultâneamente (Obs. $61 \mathrm{~m}) ; 1$ vez, sôbre as do quarto: na oitava (Obs. 62f). A Obs. $58 \mathrm{~m}$ exibe além de irregularidade na sexta meia volta distal, desvio na primeira meia volta de entrada.

Em 7 peças (figs. 41 a 47) registra-se como caracteristico comum a quase total inversão no enrolamento do cólon, eis que, as curvas concêntricas volteiam da direita para a esquerda e as excêntricas no sentido oposto, cabendo, afinal, ao derradeiro e incompleto lance de saída restabelecer o trajeto normal (Obs. $63 \mathrm{~m}, 64 \mathrm{~m}, 65 \mathrm{~m}$, 66f, 67f, 68m, 69f). As alças dêste grupo apresentam imperfeições inseridas no giro proximal, 1 vez (Obs. 66f) e nos distais, 2 vêzes (Obs. 67f, 68m).

* modlalidade não apontada por p'AIVA e borel.t.I. 


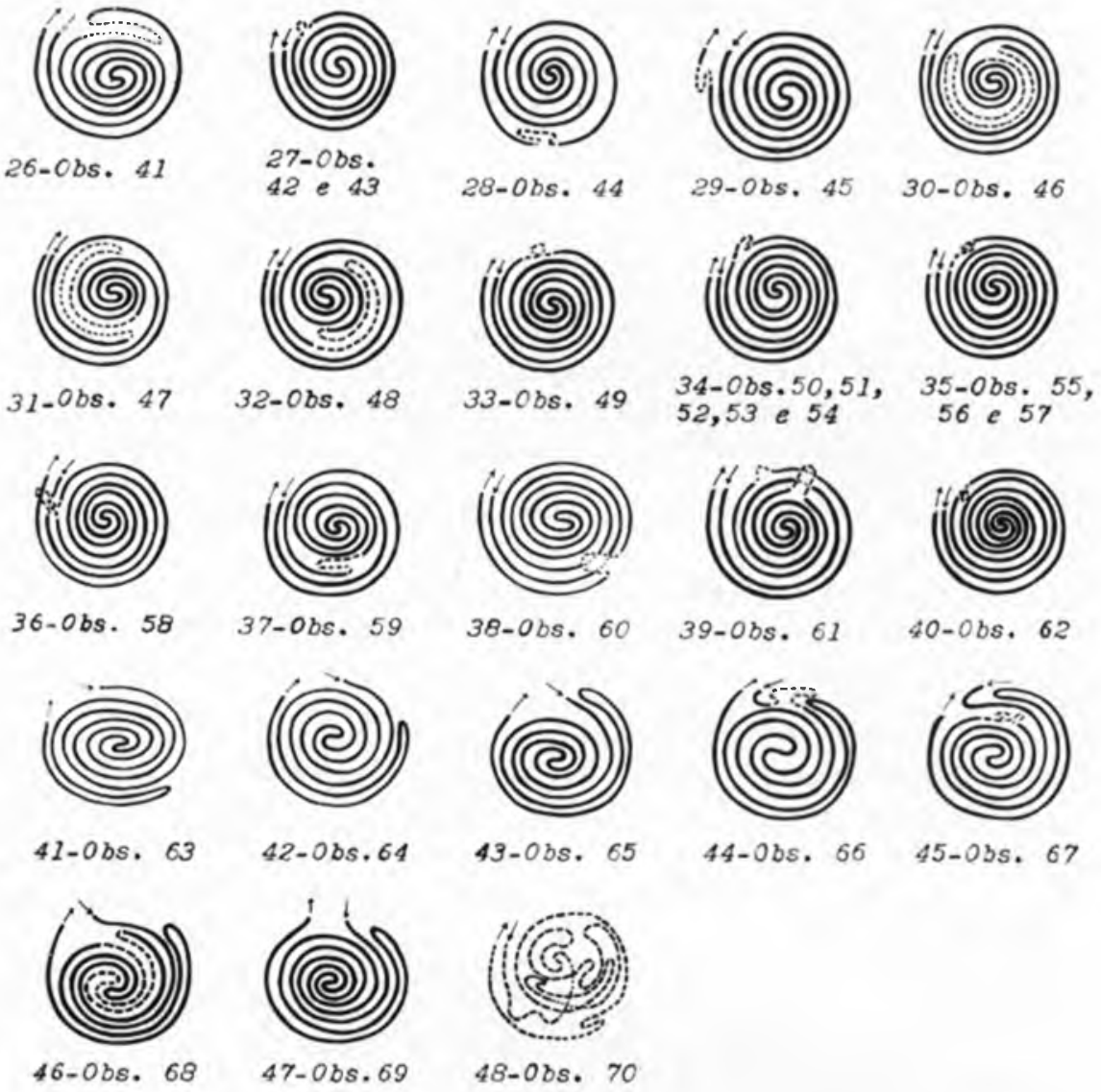

Figuras 26 a 48 - Esquemas das elisposteóes incomuns da ansa spiralis do cólon. em caprinos.

Deixamos de classificar um caso, por extremamente complicado (Obs. 70f - fig. 48).

A mudança de direção no enrodilhamento do cólon de caprinos nào é referida nas publicaçôes que tivemos a oportunidade de consultar; frise-se, todavia, que dentre nossos esquemas reconhecemos dois dos cinco de SMitil (Obs. $4 \mathrm{~m}, 31 \mathrm{~m}$ ).

Comprovamos na espécie estudada $(27,14 \%)$ o reparo de S.irTH (1955), quando chama a atençāo para o elevado número de arranjos incomuns (aproximadamente 25 'i) da última volta do cólon espiral de ovinos, isolada das restantes, fato nāo verificado nos bovinos, que a apresentam isenta de defeitos (Smith e Meadows). Parece-nos, contudo, interessante ressaltar a elevada porcentagem 
$(21,42 \%)$ de irregularidades do segmento inicial do giro de saida, trato nāo afastado do primeiro lance concêntriso.

Confrontando a freqüencia global que obtivemos $(7,39$; \pm 0,85 ), com as estabelecidas por SMITh, e por NANDA e PATEL, revelamos diferença significante apenas no segundo caso, ao nível de $5 \%$.

\section{S U M M A R Y}

Irregularities in the coiling of the ansa spiralis of the goat colon have been observed and classified. Some deviation was seen in 70 or $7.39 \%+0.85$ out of a total of 946 specimens analysed in a previous publication. These was not significant difference between sexes.

\section{REFERENCIAS BIBLIOGRÁFICAS}

ELLFNBFRGER, W. - BAUM, H. 1932 - Handbuch der vergleichenden Anatomie der Haustiere. 17. Auf. Berlin, Julius Springer.

KOLDA, J. - 1931 - Zur topographie des Darmes beim Schaf und bei der Ziege. Z. Anut. Entuickl.-Gesch., 95: 243-269.

MAR'TIN, P. - SCHAUDER, W. - 1935 - I chrbuch der Anatomie der Haustiere. $3^{4}$ Auf. Bd. 3, T. 2. Stuttgart, Schickhard \& Fbner.

NANDA. B. S. - PATEL. M. R. - 1962 - Pattern of ansa spiralis in goat colon. J. Vet. Anim. Husbundry Res., 6 (1): 29-30.

PAIVA. O. M. BORELLI, V. - 1963-64 - Disposiçōes incomuns da amsa spiralis do cólon em bovinos azchuados. Rev. Fuc. Med. vet., S. Paulo. (1): 11-17.

PAIVA. O. M. - BORELLI, V. - 1965 - Disposições da ansa spirmlis do cólon em caprinos. Folia clín. biol., S. Paulo, 34: 18-22.

SMITH, R. N. - 195.5 - Further observations on the colon primum of the sheep. J. Anat., Lond., 89 (4): 579.

SMITH. R. N. - 1958 - Irregular patterns of the ansa spiralis of the sheep colon. Brit. ret. J., 11/ 181 : 285-288.

SMI'TH. R. N. - 1959 - The arrangement of the ansa spiralis of the goat colon. Anut. Anz., 1/6 (1-5): 101-103.

SMITH, R. N. - MFADOWS, G. W. - 1956 - The arrangement of the ansa spiralis of the ox colon. J. Anat., Lond., 9') (4) : 523-526. 